\title{
The role of cytochrome $c$ oxidase subunit Va in non-small cell lung carcinoma cells: association with migration, invasion and prediction of distant metastasis
}

Wen-Liang Chen ${ }^{1,3+}$, Kuang-Tai Kuo ${ }^{2,5+}$, Teh-Ying Chou ${ }^{4,5}$, Chien-Lung Chen ${ }^{3}$, Chih-Hao Wang ${ }^{6}$, Yau-Huei Wei ${ }^{6}$ and Liang-Shun Wang ${ }^{2,7^{*}}$

\begin{abstract}
Background: Lung cancer is one of the most lethal malignancies worldwide, but useful biomarkers of lung cancer are still insufficient. The aim of this study is to identify some membrane-bound protein(s) associated with migration and invasion in human non-small cell lung cancer (NSCLC) cells.

Methods: We classified four NSCLC cell lines into high and low migration/invasion groups by Transwell and Matrigel assays. Using two-dimensional gel electrophoresis and matrix-assisted laser desorption ionization-time of flight mass spectrometry (MALDI-TOF MS), we identified 10 membrane-associated proteins being significantly overexpressed in the high migration/invasion group. The expression of the target protein in the four NSCLC cell lines was then confirmed by reverse transcription polymerase chain reaction (RT-PCR), western blot and immunostaining. RNA interference technique was applied to observe the influence of the target protein on migration and invasion. Gelatin zymography was also performed to evaluate the activities of matrix metalloproteinase (MMP)-2 and MMP-9. Expression condition of the target protein on surgical specimens was further examined by immunohistochemical staining and the clinicopathologic data were analyzed.

Results: We identified a mitochondria-bound protein cytochrome c oxidase subunit Va (COX Va) because of its abundant presence found exclusively in tumorous areas. We also demonstrated that migration and invasion of NSCLC cells decreased substantially after knocking down COX Va by siRNA. Meanwhile, we found a positive correlation between COX Va expression, BCl-2 expression and activities of MMP-2 and MMP-9 in NSCLC cells. Immunohistochemical staining of surgically resected lung adenocarcinomas in 250 consecutive patients revealed that strong COX Va expression was found in 54.8\% (137/250) of patients and correlated positively with the status of lymph node metastasis $(P=0.032)$. Furthermore, strong COX Va expression was associated with the presence of distant metastasis $(P=0.033)$.
\end{abstract}

Conclusions: Our current study showed that COX Va may play a role in migration and invasion of NSCLC cells and can be used as a biomarker to predict aggressiveness of NSCLC.

Keywords: Non-small cell lung cancer, Cytochrome c oxidase subunit Va, Migration, Invasion

\footnotetext{
* Correspondence: wangls72269@yahoo.com.tw

${ }^{\dagger}$ Equal contributors

2Division of Thoracic Surgery, Department of Surgery, Shuang Ho Hospital,

Taipei Medical University, Taipei, Taiwan

${ }^{7}$ Graduate Institute of Clinical Medicine, Taipei Medical University, Taipei,

Taiwan

Full list of author information is available at the end of the article
} 


\section{Background}

Lung cancer, predominantly non-small cell lung cancer (NSCLC), is currently the leading cause of male cancerrelated death worldwide [1]. Despite application of multimodal treatments, the overall survival of NSCLC patients remains poor [2-4]. About 40-50\% patients of NSCLC present with stage IV disease [5], and given that complete surgical resection may provide a chance of cure in patients with early-stage tumors, the reported recurrence rate in the patients with completely resected stage I NSCLC was nearly 30\% [6-8]. Moreover, among these patients with tumor recurrence, more than $70 \%$ of them have distant metastasis $[6,8]$. All these facts indicate that metastasis is the most frequently encountered problem in treating NSCLC. Metastasis is a complicated process with tumor invasion being the first step, followed by arrest in the blood stream, and finally metastatic colonization [9]. However, molecular factors that are related to invasion are still insufficient, and identification of such factors with elucidation of their molecular mechanism will provide insight into cancer biology and potentially provide new therapeutic targets for NSCLC patients.

The rapid development of proteomic technologies during the past 10 more years has brought about a massive increase in the discovery of novel cancer biomarkers. The biomarkers may have broad applications, such as for detecting the presence of a disease, monitoring the status of the disease, or evaluating the treatment response. The commonly used techniques to discover these biomarkers, also referred to as quantitative proteomics, are performed by protein separation using either two-dimensional gel electrophoresis (2DE)- or liquid chromatography (LC)-based methods coupled with protein identification using mass spectrometry (MS) [10]. Regarding lung cancer, Chen et al. used tissue samples and quantitative proteomics to identify some proteins involving in controlling gluconeogenesis and glycolysis that were associated with the survival of patients with early-stage lung adenocarcinoma [11]. Tian et al. applied NSCLC cell lines plus 2DE, matrix-assisted laser desorption ionization-time of flight mass spectrometry (MALDI-TOF MS) and tandem mass spectrometry to recognize S100A11 as an important regulatory molecule in promoting invasion and metastasis of NSCLC [12].

In the current study, we studied the non-secreted membrane-bound proteins that are associated with migration/invasion in NSCLC cells. We separated four NSCLC cell lines into high and low migration/invasion group, and then comparatively analyzed the membrane protein profiles between these two groups by conventional 2DE followed by MALDI-TOF MS. A subunit of cytochrome $c$ oxidase (COX) called COX Va was chosen to be the target of further study because of its presence exclusively in tumorous areas but not in non-tumorous areas in surgical specimens of NSCLC. The correlations between COX Va expression and activities of matrix metalloproteinase-2 (MMP-2), matrix metalloproteinase9 (MMP-9), and Bcl-2 expression were studied. The clinicopathologic significances of COX Va overexpression were also evaluated in surgically resected lung adenocarcinomas in 250 consecutive patients by immunohistochemical staining.

\section{Methods}

\section{NSCLC cell lines, cell culture and surgically dissected} tumor samples

The cell lines CL1-1 and CL1-5 were sublines with different invasiveness derived from the same parental line [13]. To increase the discrimination power of the study, we added two more NSCLC cell lines that exhibited different abilities of migration/invasion. Two human adenocarcinoma cell lines were purchased from American Type Culture Collection (ATCC) (Manassas, VA): H2126 (ATCC No. CCL-256) and H2009 (ATCC No. CRL-5911). The CL1-1 and CL1-5 were kind gifts from Professor Pan-Chyr Yang at National Taiwan University. Cells were cultured in the RPMI 1640 medium (GIBCO, Gaithersburg, MD) containing 10\% fetal bovine serum (FBS) (Jacques Boy, Reims, France), $100 \mathrm{U} / \mathrm{mL}$ penicillin (GIBCO) and $100 \mathrm{U} / \mathrm{mL}$ streptomycin (GIBCO) at $37^{\circ} \mathrm{C}$ in a $5 \% \mathrm{CO}_{2}$ atmosphere at $99 \%$ humidity. A total of 250 surgically resected tumor samples, confirmed to be adenocarcinoma by histopathologic examinations, were obtained from consecutive patients with NSCLC between 2000 and 2005. Written informed consents were obtained from the patients with the study protocol approved by the ethical committee of the hospital.

\section{Migration assay}

Cell migration assays were performed using Boyden chamber assay. The procedures were performed according to the manufacturer's recommended protocol and had been previously described [13]. Cells were harvested and suspended in RPMI 1640 medium containing 10\% FBS at a concentration of $5 \times 10^{4}$ cells $/ \mathrm{mL}$. A Transwell apparatus with $8 \mu \mathrm{m}$ pore size membrane (Millipore, Billerica, MA) was used to analyze the migration activity. The suspended cells in $100 \mu \mathrm{L}$ of serum-free RPMI 1640 medium were seeded onto the upper chamber of the apparatus and $250 \mu \mathrm{L}$ of RPMI containing 10\% FBS were added to the insert well followed by an incubation at $37^{\circ} \mathrm{C}$ for 6 hours. The inner side of the chamber was then wiped with a wet swab to remove the cells, while the outer side of the chamber was gently rinsed with PBS and subjected to Giemsa staining (Sigma-Aldrich, St. Louis, MO) for 10 minutes. The membrane was then rinsed with phosphate-buffered saline (PBS). After 
drying, the membrane was photographed followed by counting the cell number. Each cell line was evaluated in triplicate.

\section{Invasion assay}

Invasion assay was performed by a modification of the method previously described [14]. One hundred $\mu \mathrm{L}$ Matrigel (Becton Dickinson, Franklin Lakes, NJ) diluted to $1 \mathrm{mg} / \mathrm{mL}$ in serum-free RPMI was added to each upper chamber of the Transwell apparatus. After solidification of Matrigel at $37^{\circ} \mathrm{C}$, approximately $5 \times 10^{4}$ cells in serum-free RPMI were seeded onto the Matrigel over the upper chamber, followed by an addition of $250 \mu \mathrm{L}$ of RPMI containing 10\% FBS at the bottom insert-well. After incubation of the cells at $37^{\circ} \mathrm{C}$ for 18 hours, the inner side of the chamber was wiped with a wet swab to remove the cells, while the outer side of the chamber was gently rinsed with PBS and stained with the Giemsa stain solution for 10 minutes. Finally, the membrane was rinsed and allowed to air-dry. The membrane was photographed followed by counting the cell number and each cell line was evaluated in triplicate.

\section{Preparation of protein extract from membranes}

A total of $2 \times 10^{6}$ each NSCLC cells were suspended and washed by PBS for three times, followed by centrifugation at 1,500 g for 5 minutes. Cell membranes were isolated and extracted by a Compartment Protein Extraction Kit (BioChain Institute, Hayward, CA) following the procedures described by the manufacturer.

\section{D Gel electrophoresis}

Immobilized 13-cm linear $\mathrm{pH}$ gradient strips, $\mathrm{pH} 3-11$, were rehydrated overnight in a rehydration buffer containing $7 \mathrm{M}$ urea, $2 \mathrm{M}$ thiourea, $4 \%$ CHAPS, $0.5 \%$ gradient buffer and $50 \mathrm{mM}$ dithiothreitol (DTT) according to the manufacturer's instructions (Amersham Biosciences, Buckingham, UK). Isoelectrofocusing was performed at $20^{\circ} \mathrm{C}$ using an IPGphor apparatus (Amersham) with 50 $\mathrm{mA}$ for a total of $40 \mathrm{kV} / \mathrm{h}$. Strips were equilibrated in 50 $\mathrm{mM}$ Tris- $\mathrm{HCl}, 6 \mathrm{M}$ urea, 30\% (v/v) glycerol, 1\% (w/v) sodium dodecyl sulfate (SDS) and $65 \mathrm{mM}$ DTT, pH 8.8 for 20 minutes in the same buffer containing $240 \mathrm{mM}$ iodoacetamide. Equilibrated strips were transferred onto $15 \%$ uniform polyacrylamide gels poured between the two glass plates $(18 \times 20 \mathrm{~cm})$. Strips were overlaid with $0.5 \%(\mathrm{w} / \mathrm{v})$ low melting point agarose prepared in a running buffer containing bromphenol blue. Gels were run at $10^{\circ} \mathrm{C}$ using the Ettan Dalt 6 apparatus (Amersham) at $2.5 \mathrm{~W} /$ gel for 30 minutes with $30 \mathrm{~mA}$ in total until the dye front run off the bottom of the gels. All the images were collected on a Typhoon 9400 Variable Mode Imager (Amersham). Statistics and determination of protein expression levels were carried out by a Decyder software (Amersham).

\section{MALDI-TOF MS}

Protein spots were excised manually from Coomassie blue-stained gels and washed twice in $25 \mathrm{nM} \mathrm{NH}_{4} \mathrm{HCO}_{3}$ for 10 minutes until the gel pieces become transparent. Gel pieces were dehydrated with $100 \%$ acetonitrile for 5 minutes and allowed to dry, followed by incubation with $15 \mu \mathrm{L}$ of a $12.5 \mathrm{ng} / \mu \mathrm{L}$ trypsin solution at $37^{\circ} \mathrm{C}$ overnight. Trypsinized samples were then concentrated using a C18 Zip-Tips Kit (Millipore) and eluted with 50\% acetonitrile/ $0.1 \%$ trifluroacetic acid according to the manufacturer's instructions. Identification of peptides was performed using a Microflex MALDI-TOF LRF20 mass spectrometer (Bruker Daltonics, Billerica, MA). The ZipTips-purified peptide digests were spotted with a cyano4-hydroxycinnamic acid matrix and run in reflection positive-ion mode at an accelerating voltage of $25 \mathrm{kV}$. Subsequently, protein identification was done using MASCOT search engine from SWISS-PROT database (http://www.matrixscience.com).

\section{Reverse transcription polymerase chain reaction (RT-PCR)}

Level of mRNA expressions corresponding to COX Va and other genes in NSCLC cells were determined by RTPCR. Total RNA was extracted from cells using a SNAP RNA column (Invitrogen Corporation, San Diego, CA). Following spectrophotometric determination of RNA yield, cDNA is synthesized with oligo(dT) primer using avian myeloblastosis virus (AMV) reverse transcriptase. An aliquot of cDNA was subjected to 35 cycles of PCR using a standard procedure denaturing at $94^{\circ} \mathrm{C}$ for $30 \mathrm{sec}-$ onds, hybridizing at $57^{\circ} \mathrm{C}$ for 30 seconds and elongating at $72^{\circ} \mathrm{C}$ for 1 minute. Amplified products were resolved in a $1 \%$ agarose gel and visualized by ethidium bromide staining. DNA sequences of the amplified fragment were determined by an automatic sequenator (ABI, Foster City, CA) with the nucleotide sequences matched with the database from GenBank (http://www.ncbi.nlm. nih.gov/blast). The primer sequences used were as follows: COX Va (forward) 5' - TCGCCGTCATGCTGGG 3' and (reverse) 5'- CAATAAATCCTTGGGGAAGCC 3', MMP-2 (forward) 5'- AGAAGGCTGTGTTCTTTG CAG -3' and (reverse) 5' - AGGCTGGTCAGTGGCTTG -3', MMP-9 (forward) 5' - ATCCGGCACCTCTATGGTC -3' and (reverse) 5'- GCTCTGAGGGGTGGACAGT -3', Bcl-2 (forward) 5' - ATGTGTGTGGAGAGCGTCAACC -3 ' and (reverse) 5'- TGAGCAGAGTCTTCAGAGACA GCC -3'. The primer sequences for the house-keeping gene $\beta$-actin were (forward) 5'- CCAGATCATGTTT GAGACCT -3' and (reverse) 5'- CAACTAAGTCA TAGTCCGCC -3'. 


\section{Western blot}

SDS-PAGE containing 15\% polyacrylamide (unless specified otherwise) was used to analyze the COX Va content using a modified procedure described previously [15]. Electrophoresis was conducted in a vertical slab gel unit (Mini PIII, Bio-Rad, Hercules, CA) equipped with a PAC 300 power supply (Bio-Rad). All samples $(20 \mu \mathrm{g})$ for SDS-PAGE were equilibrated in $10 \mathrm{mM}$ Tris- $\mathrm{HCl}$ and $5 \%$ SDS ( $\mathrm{pH} 7.6$ ) before loading onto the gel. Following electrophoresis, the gel was soaked briefly and instantly in a transfer buffer containing $25 \mathrm{mM}$ Tris, $192 \mathrm{mM}$ glycine, $20 \%$ methanol, and $0.0375 \%$ SDS ( $\mathrm{pH} 8.3$ ) for 30 seconds. The gel was then immediately electrotransferred to a nitrocellulose membrane (Hybond-ECL extra; Amersham) at $90 \mathrm{~mA}$ for 60 minutes in a semi-dry Transfercell (Bio-Rad). The membrane was immersed in $1 \%$ skin milk for $1 \mathrm{~h}$ with gentle shaking. Following three washes with PBS for 5 minutes each, the membrane was subjected to reaction with a mouse monoclonal antibody against COX Va (MitoScience, Eugene, OR) at a 1:1,000 dilution and developed with chemiluminescence according to the method described previously [15].

\section{COX Va gene knockdown}

The small interfering RNA (siRNA) targeting human COX Va was designed using a BLOCK-iT RNAi Designer algorithm (http://rnaidesigner.invitrogen.com/rnaiexpress/). To avoid unintentional silencing of non-targeted host cell genes, sequence homology was checked using a basic local alignment search tool (BLAST) search (http://blast.ncbi. nlm.nih.gov/Blast.cgi). A scrambled-sequence oligonucleotide (CAAAGCAGGACCTCATAAGGAAATCTACC) was used as a negative siRNA control. Pairs of complementary oligonucleotides sequences were synthesized, annealed and cloned into the pRS shRNA vector (OriGene, Rockville, MD). NSCLC cells were transfected with one of the four shRNAs using TurboFect ${ }^{\mathrm{Tm}}$ (Fermentas, Glen Burnie, MD) as described below. After 48 hours, COX Va expression was assessed using RT-PCR. The results showed that two COX Va siRNA (ACTTGTTACCTATGATATGGTTCCA GAGC and CCTATGTCATCCAGGAACTTAGACC AACT) possessed the highest activity to suppress the expression of $\mathrm{COX} \mathrm{Va}$, and were used for subsequent experiments.

\section{Transfection of siRNA}

TurboFect ${ }^{\mathrm{TM}}$ was used to deliver plasmid DNA containing siRNA against human COX Va (shCOX Va) into NSCLC cells in a 24-well culture plate. In each well, approximately $5 \times 10^{4}$ cells in $1 \mathrm{~mL}$ was seeded and grown for 24 hours prior to transfection. While gently shaking, $1 \mu \mathrm{g}$ of plasmid in $100 \mu \mathrm{L}$ of RPMI medium was slowly added into $2 \mu \mathrm{L}$ of TurboFect $^{\mathrm{TM}}$ and incubated at room temperature for 15-20 minutes. The reaction mixture was then transfected into cells, which were grown in the culture medium containing $2 \mu \mathrm{g} / \mathrm{mL}$ puromycin for another 3 days, while plasmid without shRNA was used as a negative control.

\section{Gelatin zymography}

To determine the enzyme activities of MMP-2 and MMP-9, media derived from the NSCLC cells cultured for 48 hours were used for the gelatin zymography $[16,17]$. An aliquot of $10 \mu \mathrm{L}$ culture medium was loaded onto a SDS-PAGE gel under non-reducing conditions using an $8 \%$ polyacrylamide gel containing $0.1 \mathrm{mg} / \mathrm{mL}$ gelatin. After electrophoresis, the gels were washed 3 times in $2.5 \%(\mathrm{w} / \mathrm{v})$ and treated with Triton X-100 for 30 minutes at room temperature to remove the SDS and then incubated in a reaction buffer containing $50 \mathrm{mM}$ Tris (pH 7.4), $5 \mathrm{mM} \mathrm{CaCl}_{2}$ and $150 \mathrm{mM} \mathrm{NaCl}$ for 24 hours at $37^{\circ} \mathrm{C}$, followed by staining with $2.5 \%(\mathrm{w} / \mathrm{v})$ Coomassie blue in $30 \%(\mathrm{v} / \mathrm{v})$ methanol and $10 \%(\mathrm{v} / \mathrm{v})$ acetic acid. The MMP-2 and MMP-9 activities were detected as clear bands on a blue background with stained undigested gelatin.

\section{Immunocytochemical staining of cultured cells}

Glass cover slips were sterilized by dipping them in $90 \%$ ethanol. After drying over a flame, each slip was placed into each well of 6-well culture plates. NSCLC cells $\left(5 \times 10^{6}\right)$ in $2 \mathrm{~mL}$ of RPMI medium containing $10 \%$ FBS were seeded over the slip and grown at $37^{\circ} \mathrm{C}$ until $50-70$ $\%$ confluence reached. Following removal of the culture medium, cells in each well were rinsed twice with PBS and fixed with $3 \%$ paraformaldehyde (Sigma) in PBS and then incubated at room temperature for 20 minutes. After rinses with PBS for three times, cells were treated with a Triton X-100 buffer containing $50 \mathrm{mM}$ Tris, 1 $\mathrm{mM}$ EDTA and $0.5 \%$ Triton $\mathrm{X}-100, \mathrm{pH} 8.0$ at room temperature for 15 minutes. Following washes with PBS, the slips were incubated with $10 \%$ normal horse serum to block nonspecific staining and then incubated with a mouse monoclonal antibody against COX Va (MitoScience, Eugene, OR) at a 1:100 dilution in a humid chamber at $4^{\circ} \mathrm{C}$ overnight. After washes, cells were incubated with a biotinylated horse anti-mouse IgG for 30 minutes and incubated with streptavidinconjugated peroxidase for 30 minutes. Cells containing COX Va were visualized by incubation with 3,3-diaminobenzidine solution $(0.3 \%$ hydrogen peroxide and $0.05 \%$ 3,3-diaminobenzidine) and counterstained with hematoxylin. Negative controls were prepared by substituting a normal mouse IgG as the primary antibody.

\section{Preparation of tissue core array}

All of the pathologic sections were reviewed by one pathologist (T.Y.C.). Cores of tissue in demand were 
manually obtained from a tissue block by a 10-gauge syringe needle as described previously [18]. The cores were placed in a warm cast containing partially melted paraffin and arranged into a $6 \times 8$ array with one core at the upper-left corner removed as an orientation marker. Each array contained 47 cores with each patient sample from two to five cores, depending on the availability of tissue blocks. The positions of each core were recorded on reference sheets to facilitate data acquisition.

\section{Immunohistochemical staining of tumor tissues and evaluation of COX Va immunostaining}

Immunostaining was performed by biotin-streptavidinperoxidase procedures using a Vectastain ABC Kit (Vector Laboratories, Burlingame, CA). Fresh tumor tissues from patients with NSCLC and tissue core arrays were fixed in formalin and embedded in paraffin, to which sliced serial sections in $4 \mu \mathrm{m}$ were mounted on glass slides, deparaffinized with xylene and dehydrated with alcohol according to the manufacturer's instructions. Following retrieval of the antigen by heating the slice in a microwave for 2 minutes at $900 \mathrm{~W}$, the slides were incubated with $0.3 \% \mathrm{H}_{2} \mathrm{O}_{2}$ solution in methanol for 30 minutes to block endogenous peroxidase. After rinses with PBS for three times, the tissues were treated with protease $\mathrm{K}(20 \mu \mathrm{g} / \mathrm{mL}$ a buffer containing $50 \mathrm{mM}$ Tris, 1mM EDTA, 0.5\% Triton X-100, pH 8.0) for 15 minutes at room temperature to expose the immunoreactive parts of $\mathrm{COX} \mathrm{Va}$ on the mitochondrial membrane. Thereafter, the rest of the immunostaining procedures were conducted according to that described above.

Each stained core was evaluated according to the gross percentage of cells demonstrating nuclear and cytoplasmic immunoreactivity on the tumor part, and the assessment was accomplished independently by two examiners (W.L. $\mathrm{C}$ and K.T.K.). The scoring criteria for COX Va were as follows: 0 , no appreciable staining in cells; $1+$, appreciable nuclear staining with cytoplasmic staining in $<25 \%$ of cells; $2+$, appreciable nuclear staining with cytoplasmic staining between $25 \%$ and $50 \%$ of cells; $3+$ : appreciable nuclear staining with cytoplasmic staining in $>50 \%$ of cells. Only $3+$ staining was defined as COX Va
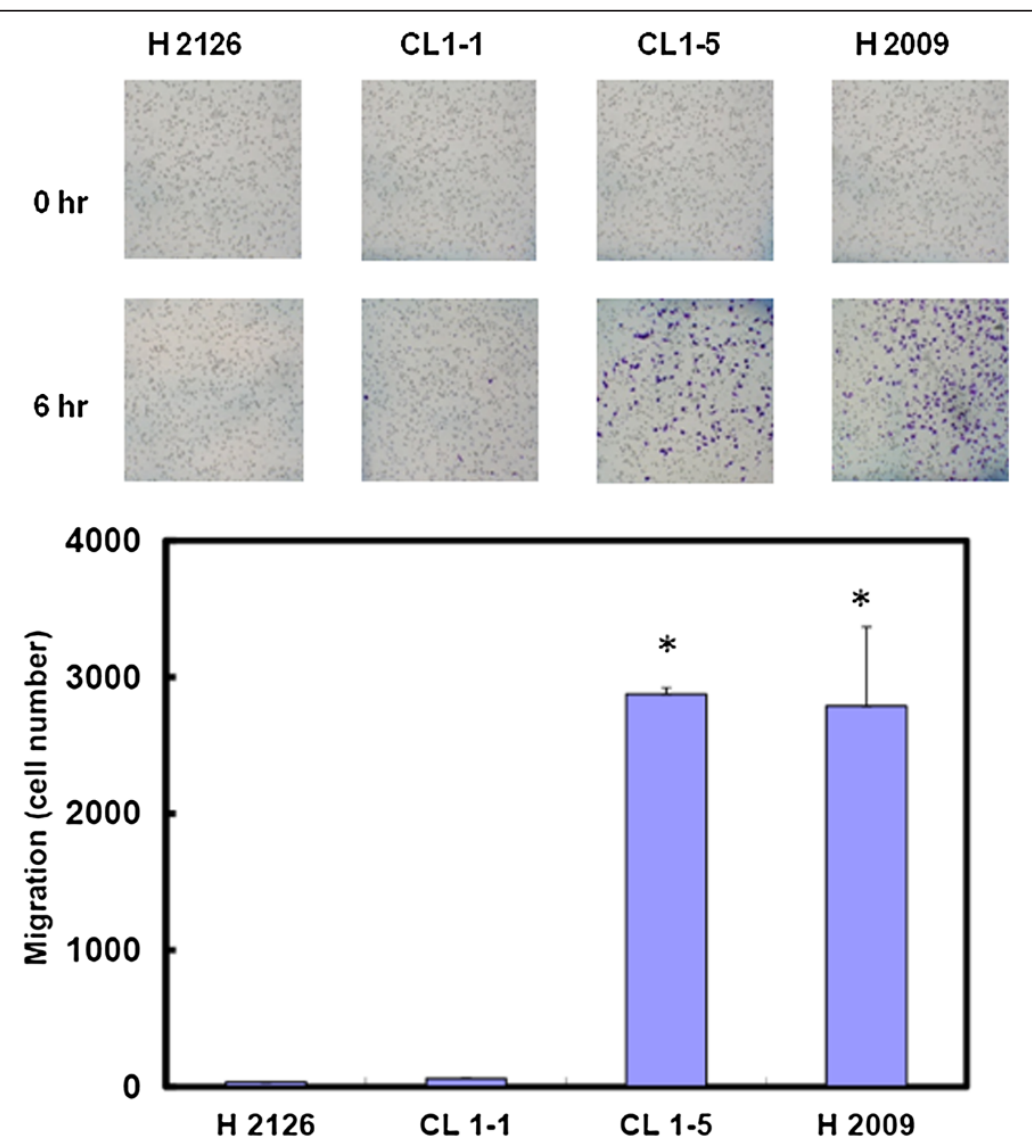

Figure 1 Differences in migration ability among four NSCLC cell lines (H2126, CL1-1, CL1-5 and H2009). Migration ability was assessed by Transwell assay. Each bar represents the mean \pm SD (standard deviation) of the results from triplicates. The asterisk indicates a significant difference $(P<0.001)$ between either H2009 or CL1-5 and CL1-1 or H2009. 
overexpression or COX Va (+). Stage of disease progression was classified according to the 6th edition American Joint Committee on Cancer (AJCC) Staging System.

\section{Statistical analysis}

The data were analyzed and are presented as mean \pm SD (standard deviation). The difference between the compared groups was calculated by Mann-Whitney U test. Pathologic T, N, M status and tumor stage derived from new lung cancer staging system in the database were reviewed. The relationship between pathologic factors and COX Va expression status was analyzed by $x^{2}$ test (or two-tailed Fisher's exact test when the expected number in any cell was smaller than 5 cases). A $P$ value $<0.05$ is considered statistically significant.

\section{Results}

Migration and invasion

We evaluated four NSCLC cell lines (CL1-1, CL1-5, H2126 and H2009) for their abilities in cell migration and invasion. As shown in Figures 1 and 2, those cell lines had stronger migration also had stronger invasion.
Thus we divided them into low (H2126 and CL1-1) and high (CL1-5 and H2009) migration/invasion group.

\section{D Gel electrophoresis analysis}

Next, we analyzed the membrane protein profiles between the high and low migration/invasion groups by 2D PAGE. A representative example of the comparison is depicted in Figure 3. The proteins overexpressed in high migration/invasion group were selected as targets since they might serve as potential biomarkers in a usual pathology laboratory. Following MALDI-TOF MS analyses, we identified 10 proteins, with their details listed in Table 1. Among them, one mitochondrial membranebound protein called cytochrome $c$ oxidase subunit $\mathrm{Va}$ (COX Va), was of most interest because it was markedly overexpressed in tumorours tissues.

\section{Characterization of molecular and protein expression of COX Va in NSCLC cell lines by RT-PCR, western blot and immunocytochemical staining}

To evaluate whether this newly identified protein COX Va was overexpressed in situ in the cells with high migration/

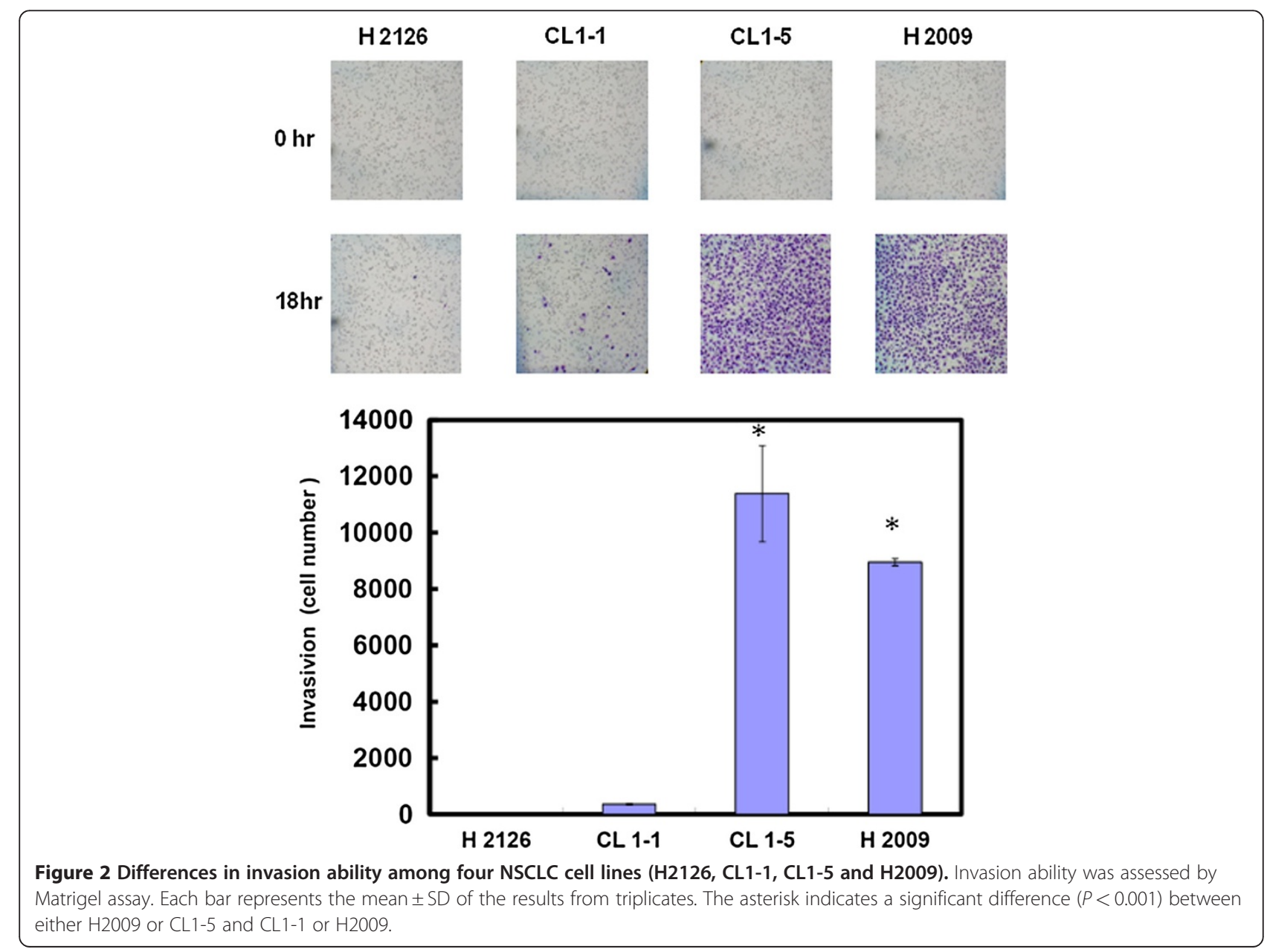




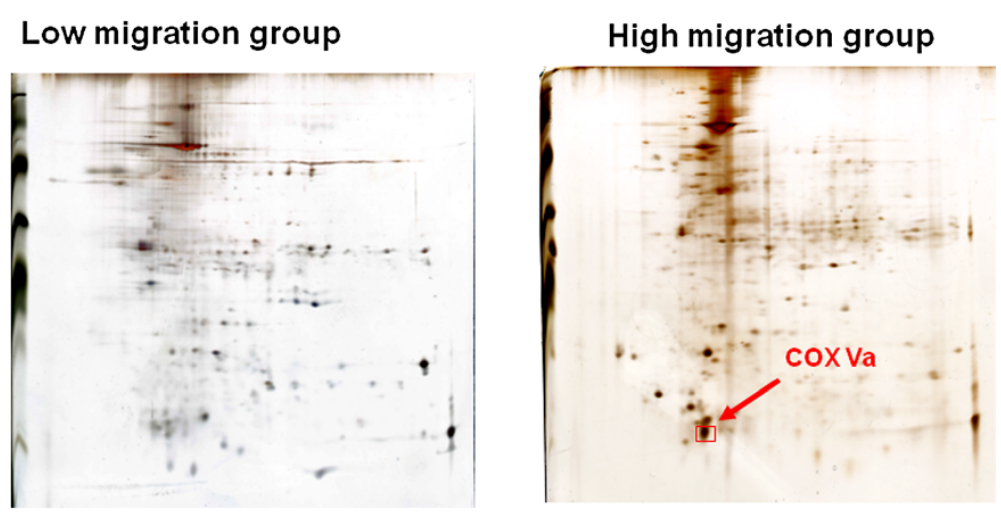

Figure 3 A typical example of membrane-associated proteins analyzed by 2D-gel between low (CL1-1) and high (CL1-5) migration/ invasion lung cancer cell lines. A sample containing $200 \mu \mathrm{g}$ of membrane proteins was initially run by isoelectrofocusing (pH 3 to 10 from left to right), which was followed by a conventional SDS-PAGE. Gels were then stained with a silver staining solution.

invasion, we conducted analysis for its mRNA expression using RT-PCR. Figure 4A shows that the cell lines in high migration/invasion group had greater COX Va mRNA levels as compared with those in low migration/invasion group. The higher expression in protein level was also documented by western blot, as shown in Figure 4B. To further confirm these observations, we determined the level of COX Va using an immunostaining in these four cell lines. Pre-immune serum or unrelated monoclonal antibody was used as a negative control. After treatment of the cells with Triton X-100, Figure 4C shows that there was a striking difference in immunostaining activity between the high and low migration/invasion cells. These results confirmed that the cell lines in high migration/invasion group had higher intensity of COX Va expression.

\section{Decreased migration/invasion of NSCLC cells by knocking down COX Va}

To further testify whether COX Va plays a role in tumor cell migration/invasion, we constructed shRNA to knockdown COX Va at its mRNA level in the high migration/invasion group, with the $\mathrm{H} 2009$ cells as the representative. The result of RT-PCR (Figure 5A) shows that the expression of COX Va mRNA was substantially attenuated in COX Va-knockdown cells comparing with original H2009 (control) or scramble. Furthermore, cells with COX Va-knockdown had $42 \%$ decrease in migration and 52\% decrease in invasion, respectively, as compared with original H2009 (control) or scramble $(P<0.01)$ (Figure 5B and $5 \mathrm{C})$. These findings suggest that expression of COX Va was associated with migration/invasion of NSCLC cells.

\section{MMP assay}

To look into the factors that were possibly linked to COX Va expression, we examined the expression of MMP-2 and MMP-9 in these four NSCLC cells. In addition to RT-PCR, we used gelatin zymography to assess the enzyme expression of MMP-2 and MMP-9. Figure $6 \mathrm{~A}$ and $6 \mathrm{~B}$ show that cell lines with high

Table 1 Up-regulated proteins in highly migration/invasion NSCLC cell lines

\begin{tabular}{|c|c|c|c|}
\hline Protein name & Molecular weight $(\mathrm{kDa})$ & Location & Function \\
\hline COX Va & 16.7 & mitochondria inner membrane & electron carrier activity \\
\hline Caveolin-1 & 25 & membrane & $\begin{array}{l}\text { protein Tyrosine Phosphatase and } \\
\text { G-protein-mediated signal transduction }\end{array}$ \\
\hline Galectin-1 & 14 & membrane & $\begin{array}{c}\text { regulate apoptosis, cell proliferation and } \\
\text { differentiation }\end{array}$ \\
\hline Galectin-3 & 35 & cytosol and Mitochondria inner membrane & cell differentiation \\
\hline Glucose-regulated protein 78 & 78 & endoplasmic reticulum & $\begin{array}{l}\text { involved in the folding and assembly } \\
\text { of proteins }\end{array}$ \\
\hline Heat shock 70 kDa protein 8 & 70 & endoplasmic reticulum & protein refolding and antiapoptosis, \\
\hline Tyrosine-protein kinase 7; AXL & 107 & membrane & signal transducer \\
\hline Trophinin & 70 & membrane & adhesion molecule \\
\hline UDP-glucuronosyltransferase & 56 & endoplasmic reticulum & bilirubin conjugation \\
\hline Vimentin & 57 & cytoskeleton & cellular component movement \\
\hline
\end{tabular}




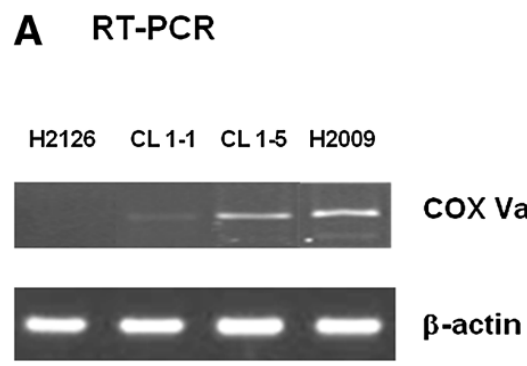

B Western blot

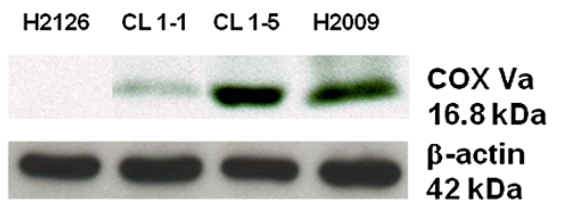

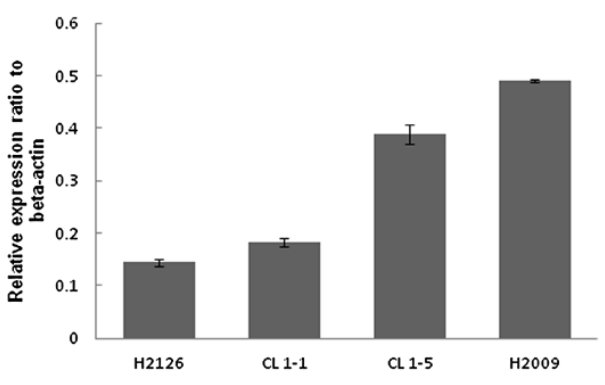

C Immunostaining
H 2126

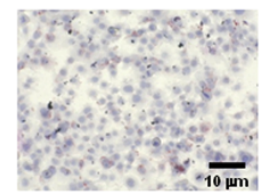

CL 1-5

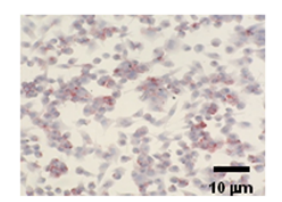

CL 1-1

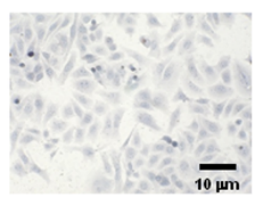

H 2009

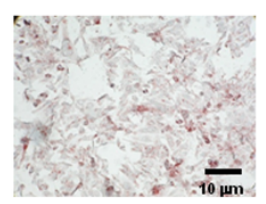

Figure 4 Comparison of the expression of mRNA and protein of COX Va in NSCLC cell lines using RT-PCR, western blot and

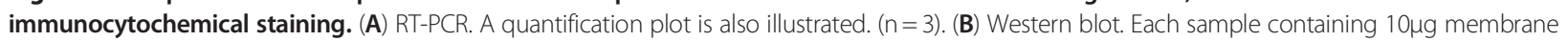
proteins was loaded on a 15\% SDS-PAGE. (C) Immunocytochemical staining was performed by using a monoclonal antibody against COX Va.

migration/invasion (CL1-5 and H2009) had stronger expression of MMP-2 and MMP-9 comparing to those with low migration/invasion (H2126 and CL1-1), in mRNA and enzyme activity level, respectively. These findings may partly account for why CL1-5 and H2009 had higher migration/invasion than did H2126 and CL1-1. Furthermore, the enzyme activity of MMP-2 and MMP-9 was also shown to be reduced in COX Va knockdowned H2009 cells (Figure 6C).

\section{Bcl-2 evaluation}

$\mathrm{Bcl}-2$ and its family proteins are integral membrane proteins located mainly on the outer membrane of mitochondria and have been reported to interact with cytochrome $c[19,20]$. Therefore, the mRNA expression of Bcl-2 in four cell lines was also evaluated. As shown in Figure 7A, the cell lines in high migration/invasion group (CL1-5 and H2009) had higher expression of Bcl2 mRNA. After knocking down COX Va expression, the H2009 cells with COX Va-knockdown also had lower expression of Bcl-2 mRNA (Figure 7B).

\section{Expression of COX Va in surgically resected tissues of patients with NSCLC}

Paired tumorous and non-tumorous (stromal area) fresh frozen specimens from 10 subjects with NSCLC were randomly selected to evaluate the mRNA expression of COX Va by RT-PCR. It revealed that the expression condition of COX Va mRNA was variable, with greater level in tumorous area in most cases (Figure 8A). With respect to immunostaining in the fresh resected tissues, it was of interest to note that COX Va can only be stained by treating the tumorous tissue with Triton X-100 plus protease $\mathrm{K}$. A typical example showing tumorous area being highly positive is depicted in Figure 8B. To our surprise, we found that non-tumorous areas were essentially negative for COX Va, which is similar to negative controls stained with normal mouse serum (data not shown). However, it should be reminded that areas without reactivity in immunostaining do not necessarily indicate a lack of biosynthesis of COX Va. For example, expression of COX Va mRNA in non-tumorous tissue could still be observed in subject No.3, 4 and 8 (Figure 8A).

Using tissue core array, we also studied the immunoreactivities of COX $\mathrm{Va}$ in formalin-fixed, paraffinembedded specimens and analyzed their pathologic significance in 250 consecutive patients with adenocarcinoma. Strong COX Va expression was found in 54.8\% (137/ 250) of patients and correlated positively with the $\mathrm{N}$ status $(P=0.032)$, but without significant correlation with $\mathrm{T}$ status $(P=0.667), M$ status $(P=0.802)$ or stage $(P=0.232)$ (Table 2). During follow-up, strong COX Va expression 


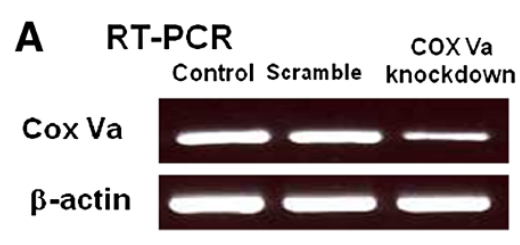

B Migration assay
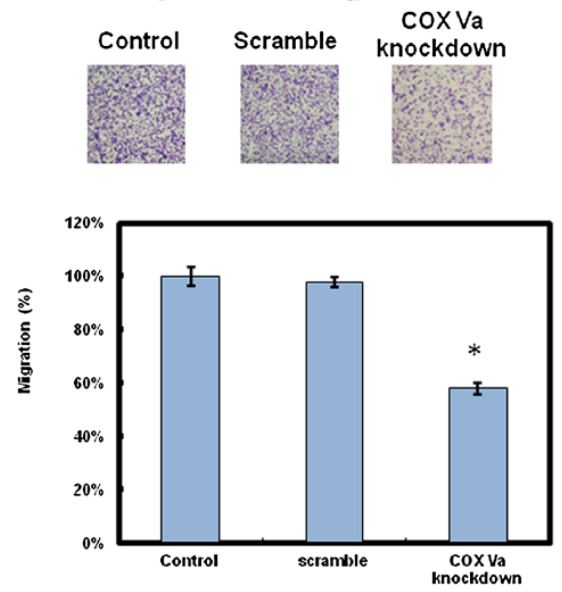

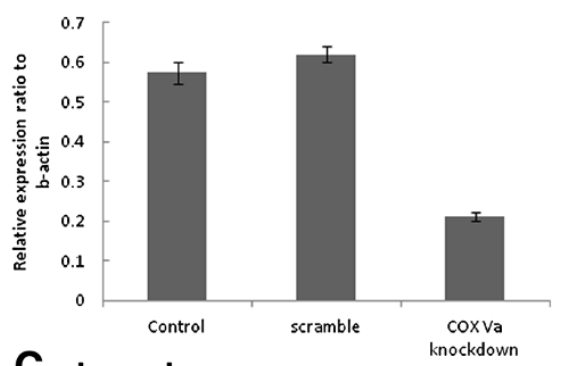

C Invasion assay
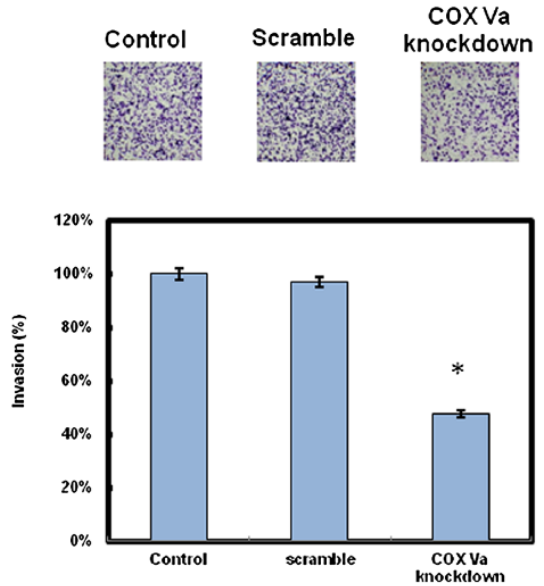

Figure 5 The effect of COX Va gene knockdown on the migration/invasion of NSCLC cells. (A) RT-PCR for COX Va in original H2009 cells (control), scramble and $\mathrm{H} 2009$ cells transfected with the shRNA against human COX Va. A quantification plot is also illustrated. $(n=3)$. (B) Migration activity of $\mathrm{H} 2009$ cells following transfection. Each bar represents the mean \pm SD of the results from triplicates. (C) Invasion activity of $\mathrm{H} 2009$ cells following transfection. Each bar represents the mean \pm SD of the results from triplicates. The asterisk indicates a significant difference $(P<0.01$ ) between either original H2009 (control) or scramble and COX Va-knockdown cells.

A

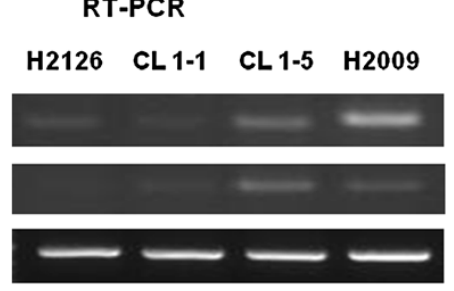

B

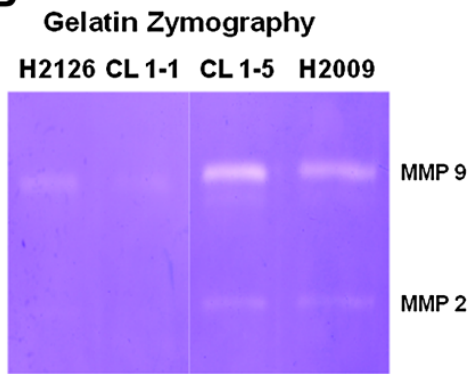

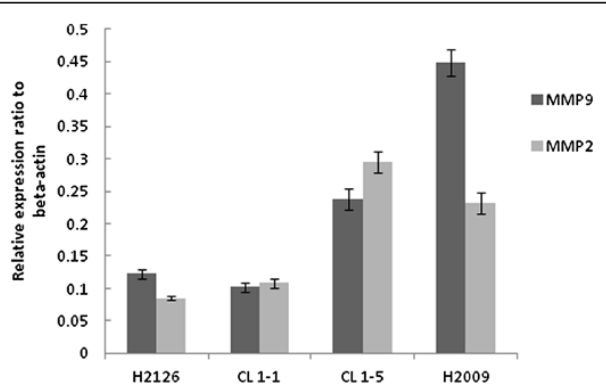

C Control Scramble $\underset{\text { knockdown }}{\operatorname{coxva}}$

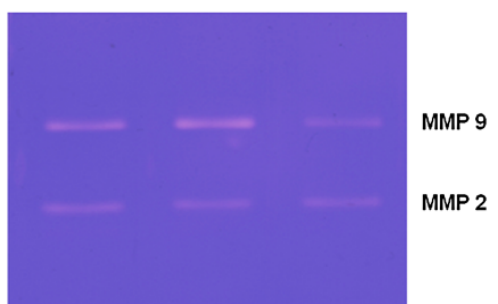

Figure 6 Studies of MMP-2 and MMP-9 in NSCLC cells. (A) RT-PCR for MMP-2 and MMP-9 in four NSCLC cell lines. A quantification plot is also illustrated. $(n=3)$. (B) Gelatin zymography of enzyme activity determined from concentrated culture media of four NSCLC cell lines after 48 hours of incubation. (C) MMP-2 and MMP-9 activities in original H2009 cells (control), scramble and COX Va-knockdown H2009 cells. 
A

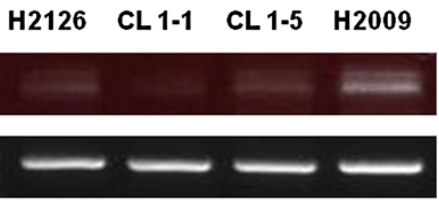

Bcl-2

$\beta$-actin

B

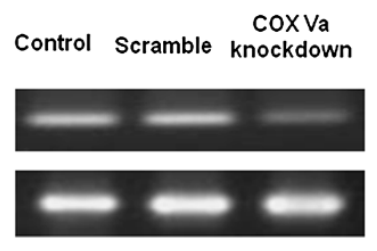

Bcl-2

$\beta$-actin
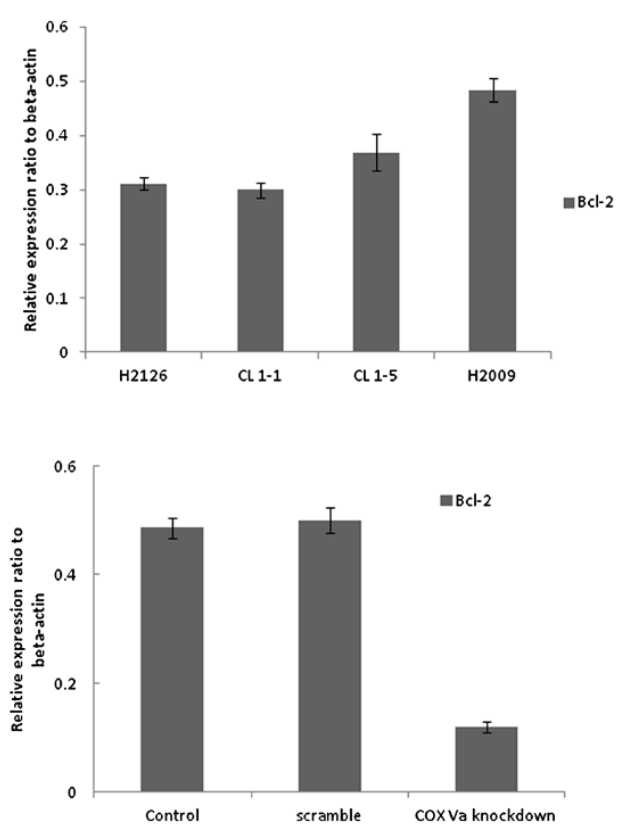

Figure 7 Bcl-2 expression in NSCLC cells. (A) RT-PCR for BCl-2 in four NSCLC cell lines. A quantification plot is also illustrated. ( $n=3)$. (B) RT-PCR for Bcl-2 in original H2009 cells (control), scramble and COX Va-knockdown H2009 cells. A quantification plot is also illustrated. $(n=3)$.

was associated with distant metastasis significantly $(P=0.033)$ but not local recurrence $(P=0.465)$. However, COX Va expression was not associated with either overall survival $(P=0.329)$ or disease-free survival $(P=0.189)$. The median survival was 65.1 months for strong COX Va patients, and 69.8 months for weak COX Va patients.

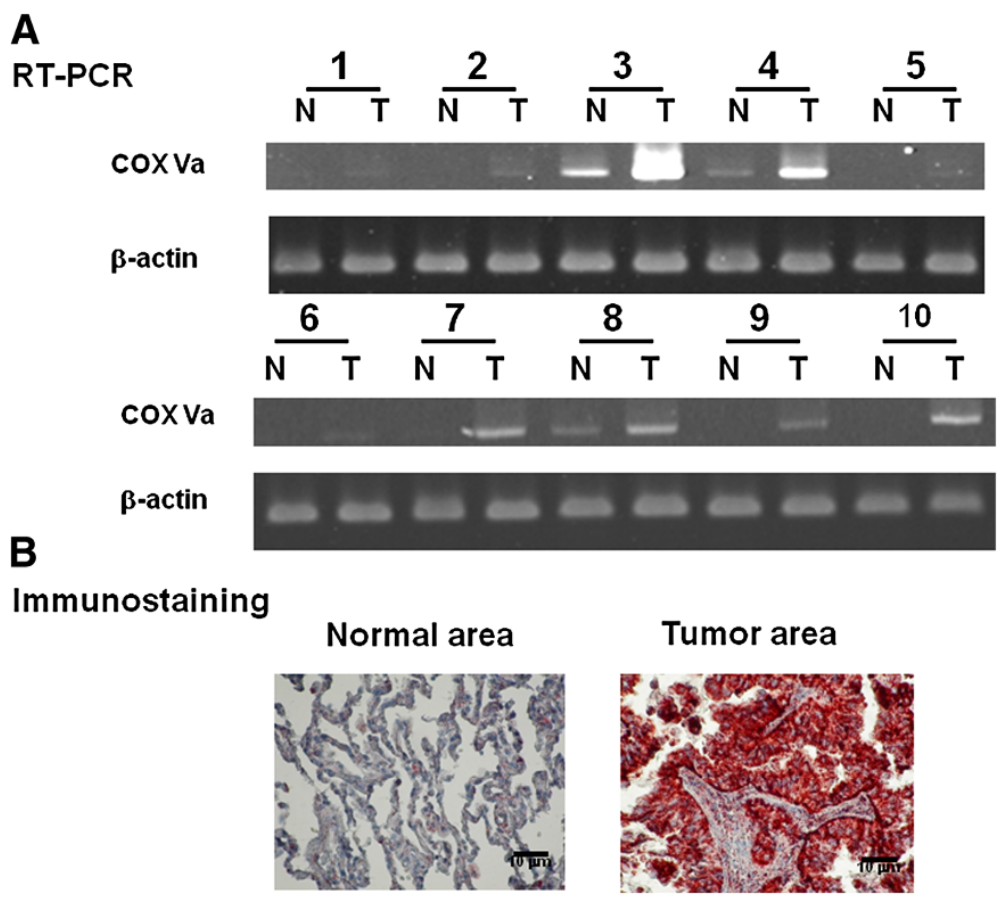

Figure 8 Molecular expression and immunostaining of COX Va in pairs of surgically resected lung tissues from 10 patients with NSCLC. (A) RT-PCR. (B) Immunohistochemical staining. A typical example depicts a positive immunostaining in tumorous tissues (right), but not in non-tumorous tissues (left). 


\begin{tabular}{|c|c|c|c|c|}
\hline \multicolumn{2}{|l|}{ Parameters } & $\begin{array}{c}\text { COX Va }(+) \\
n=137\end{array}$ & $\begin{array}{c}\text { COX Va }(-) \\
n=113\end{array}$ & $p$ value \\
\hline \multicolumn{2}{|l|}{ T status } & & & 0.667 \\
\hline \multicolumn{2}{|l|}{$\mathrm{T} 1$} & 27 & 31 & \\
\hline \multicolumn{2}{|l|}{$\mathrm{T} 2$} & 98 & 68 & \\
\hline \multicolumn{2}{|l|}{ T3 } & 1 & 5 & \\
\hline \multicolumn{2}{|l|}{$\mathrm{T} 4$} & 11 & 9 & \\
\hline \multicolumn{2}{|l|}{$\mathrm{N}$ status } & & & $0.032^{*}$ \\
\hline \multicolumn{2}{|l|}{$N(-)$} & 83 & 83 & \\
\hline \multicolumn{2}{|l|}{$N(+)^{a}$} & 54 & 30 & \\
\hline \multicolumn{2}{|l|}{ M status } & & & 0.802 \\
\hline \multicolumn{2}{|l|}{$M(-)$} & 130 & 108 & \\
\hline \multicolumn{2}{|l|}{$M(+)$} & 7 & 5 & \\
\hline \multicolumn{2}{|l|}{ Stage } & & & 0.232 \\
\hline \multicolumn{2}{|l|}{ I } & 76 & 72 & \\
\hline \multicolumn{2}{|l|}{$\|$} & 15 & 9 & \\
\hline \multicolumn{2}{|l|}{ III } & 39 & 27 & \\
\hline \multicolumn{2}{|l|}{ IV } & 7 & 5 & \\
\hline Local & yes & 9 & 5 & 0.465 \\
\hline recurrence & no & 128 & 108 & \\
\hline Distant & yes & 25 & 10 & $0.033^{*}$ \\
\hline metastasis & no & 112 & 103 & \\
\hline
\end{tabular}

${ }^{\mathrm{a}} \mathrm{N}(+)$ includes $\mathrm{N} 1$ and $\mathrm{N} 2$ but without $\mathrm{N} 3$.

\section{Discussion}

Metastasis remains the major problem in managing NSCLC, and invasion is the first step of metastasis. Thus understanding more about invasion will provide us greater opportunities to treat NSCLC. Previous reports [21,22] have concluded some markers regarding metastasis and invasion in lung cancer, such as MMPs, vascular endothelial growth factor (VEGF) and cyclooxygenase-2 (COX-2). Among these markers, some therapeutic agents derived from them had been proved to be useful in clinical practice [23-25].

In this study, using NSCLC cell lines with different abilities of migration/invasion, we distinguished a membrane-bound protein that was associated with migration/invasion of NSCLC by proteomic approach. Totally 10 membrane-associated proteins were identified after 2D gel electrophoresis and subsequent MALDITOF MS studies, and COX Va was of particular interest because of its exclusive presence in the surgical specimens from NSCLC patients. Further experiments supported the assumption that COX Va was associated with migration/invasion of NSCLC.

First, knockdown of COX Va gene expression suppressed migration/invasion of NSCLC cells effectively (Figure 5). Second, the cells expressed higher level of
COX Va also had higher expressions and activities of MMP-2 and MMP-9 (Figure 6A and 6B), which had been reported to be related to migration and invasion in different types of tumor cells [16,17,26-28]. Meanwhile, such a correlation was confirmed again in COX Va knockdown study (Figure 6C). Third, in 250 surgically resected adenocarcinomas, overexpression of $\mathrm{COX} \mathrm{Va}$ correlated positively with the $\mathrm{N}$ status (lymph node metastasis) and distant metastasis. These data highlighted the clinicopathologic role of COX Va.

Structurally, COX is a mitochondrial transmembrane enzyme consisting of 13 subunits in mammals, among which only the three largest subunits (I-III) are encoded by mitochondrial DNA. COX is a rate-limiting enzyme present as a dimeric unit participating at the terminal step in respiratory chain to reduce molecular oxygen into water and pump protons across the inner mitochondrial membrane [29-32]. A recent study showed that high levels of COX activity and mitochondrial respiration in tumor cells lead to an overexpression of Bcl2 [33]. Furthermore, it has been reported that Bcl-2 directly binds to COX to promote survival of cancer cells by maintaining a slight pro-oxidant state through elevated mitochondrial respiration during basal conditions [34]. In the same study, a protein-protein interaction between COX Va and Bcl-2 was also documented by coimmunoprecipitation.

Although the causal relationship between COX Va and MMP-2/MMP-9 could not be demonstrated in the current study, some speculation may be provided. From our current data, the cell lines in high migration/invasion group (CL1-5 and H2009) also had higher expression of Bcl-2 and knocking down COX Va was associated with decreased expression of Bcl-2. It implied that the activity of COX Va was parallel to the expression of Bcl-2. Previously, it has been reported that Bcl-2 promotes invasion and lung metastasis by inducing MMP-2 in NSCLC cells [35] and coexpression of Bcl-2 and N-Myc induces MMP-2 secretion and activity in human neuroblastoma cells [36]. In an animal study, down-regulation of Bcl-2 was observed to be associated with down-regulation of both MMP-2 and MMP-9 expression [37]. Thus we consider that overexpression of COX Va may result in increased expression of MMP-2 and MMP-9 via collaboration with Bcl-2. Nevertheless, further investigations are needed to elucidate the mechanistic insight.

\section{Conclusions}

To sum up, we identified a membrane-bound protein COX Va from NSCLC cells with different abilities of migration/invasion and confirmed its relationship to migration/invasion by several in vitro experiments. The clinicopathologic role of COX Va was also demonstrated 
by its positive correlation with lymph node metastasis in surgically resected adenocarcinomas plus prediction of distant metastasis during follow-up. Meanwhile, expression of COX Va was found parallel to expression of Bcl2, MMP-2 and MMP-9. All these data suggested that COX Va plays a role in migration/invasion of NSCLC cells and is worth of further study to serve as a therapeutic target in NSCLC.

\section{Competing interests}

The authors declare that they have no competing interests.

\section{Acknowledgments}

The authors appreciate the critical review and comments from Dr. Simon JT Mao during the preparation of this manuscript, and prize the contribution of Dr. Yu-Chung Wu for his assistance in collecting clinical data. They would also like to thank Ms. Li-Shu Chao and Jhih-Yi Teng for their excellent technical assistance. This work was supported by the National Science Council of Taiwan, Republic of China (grants 99-2314-B-009-001, 96-2628-B -385-001, 96-2314-B-385-001 and 98-2314-B-038-030).

\section{Author details}

'Department of Biological Science and Technology, National Chiao Tung University, Hsinchu, Taiwan. ${ }^{2}$ Division of Thoracic Surgery, Department of Surgery, Shuang Ho Hospital, Taipei Medical University, Taipei, Taiwan. ${ }^{3}$ Translational Research Laboratory, Shuang Ho Hospital, Taipei Medical University, Taipei, Taiwan. ${ }^{4}$ Department of Pathology, Taipei Veterans General Hospital, Taipei, Taiwan. ${ }^{5}$ Institute of Clinical Medicine, National Yang-Ming University, Taipei, Taiwan. ${ }^{6}$ Institute of Biochemistry and Molecular Biology, National Yang-Ming University, Taipei, Taiwan. ${ }^{7}$ Graduate Institute of Clinical Medicine, Taipei Medical University, Taipei, Taiwan.

\section{Authors' contributions}

WLC initiated the study, performed proteomic part of the experiments and drafted the manuscript. KTK performed the immunohistochemistry part of the experiments and assisted in the writing of the manuscript. CLC performed migration and invasion parts of the experiments. CHW, TYC and YHW contributed to experimental design and data discussion. LSW designed and supervised the experiments, and proofed the manuscript. All authors read and approved the final draft of the manuscript.

Received: 17 November 2011 Accepted: 29 June 2012

Published: 29 June 2012

\section{References}

1. Jemal A, Bray F, Center MM, Ferlay J, Ward E, Forman D: Global cancer statistics. CA Cancer J Clin 2011, 61:69-90.

2. Farray D, Mirkovic N, Albain KS: Multimodality therapy for stage III nonsmall-cell lung cancer. J Clin Oncol 2005, 23:3257-3269.

3. Reed MF, Molloy M, Dalton EL, Howington JA: Survival after resection for lung cancer is the outcome that matters. Am J Surg 2004, 188:598-602.

4. Schiller JH, Harrington D, Belani CP, Langer C, Sandler A, Krook J, Zhu J, Johnson DH: Eastern Cooperative Oncology Group: Comparison of four chemotherapy regimens for advanced non-small-cell lung cancer. N Engl J Med 2002, 346:92-98.

5. Hoffman PC, Mauer AM, Vokes EE: Lung cancer. Lancet 2000, 355:479-485.

6. Martini N, Bains MS, Burt ME, Zakowski MF, McCormack P, Rusch WW, Ginsberg RJ: Incidence of local recurrence and second primary tumors in resected stage I lung cancer. J Thorac Cardiovasc Surg 1995, 109:120-129.

7. Kawachi R, Tsukada H, Nakazato Y, Takei H, Furuyashiki G, Koshi-ishi Y, Goya $\mathrm{T}$ : Early recurrence after surgical resection in patients with pathological stage I non-small cell lung cancer. Thorac Cardiovasc Surg 2009, 57:472-475.

8. Hung JJ, Hsu WH, Hsieh CC, Huang BS, Huang MH, Liu JS, Wu YC: Postrecurrence survival in completely resected stage I non-small cell lung cancer with local recurrence. Thorax 2009, 64:192-196.

9. Steeg PS: Tumor metastasis: mechanistic insights and clinical challenges. Nat Med 2006, 12:895-904.
10. Wong SC, Chan CM, Ma BB, Lam MY, Choi GC, Au TC, Chan AS, Chan AT: Advanced proteomic technologies for cancer biomarker discovery. Expert Rev Proteomics 2009, 6:123-134.

11. Chen G, Gharib TG, Wang H, Huang CC, Kuick R, Thomas DG, Shedden KA, Misek DE, Taylor JM, Giordano TJ, Kardia SL, lannettoni MD, Yee J, Hogg PJ, Orringer MB, Hanash SM, Beer DG: Protein profiles associated with survival in lung adenocarcinoma. Proc Natl Acad Sci U S A 2003, 100:13537-13542.

12. Tian T, Hao J, Xu A, Hao J, Luo C, Liu C, Huang L, Xiao X, He D: Determination of metastasis-associated proteins in non-small cell lung cancer by comparative proteomic analysis. Cancer Sci 2007, 98:1265-1274.

13. Soel SM, Choi OS, Bang MH, Yoon Park JH, Kim WK: Influence of conjugated linoleic acid isomers on the metastasis of colon cancer cells in vitro and in vivo. J Nutr Biochem 2007, 10:650-657.

14. Chu YW, Yang PC, Yang SC, Shyu YC, Hendrix MJ, Wu R, Wu CW: Selection of invasive and metastatic subpopulations from a human lung adenocarcinoma cell line. Am J Respir Cell Mol Biol 1997, 17:353-360.

15. Chen WL, Liu WT, Yang MC, Hwang MT, Tsao JH, Mao SJ: A novel conformation-dependent monoclonal antibody specific to the native structure of beta-lactoglobulin and its application. J Dairy Sci 2006, 89:912-921.

16. Jovanović M, Stefanoska I, Radojcić L, Vićovac L: Interleukin-8 (CXCL8) stimulates trophoblast cell migration and invasion by increasing levels of matrix metalloproteinase (MMP)2 and MMP9 and integrins alpha5 and beta1. Reproduction 2010, 139:789-798.

17. Hung WC, Tseng WL, Shiea J, Chang HC: Skp2 overexpression increases the expression of MMP-2 and MMP-9 and invasion of lung cancer cells. Cancer Lett 2010, 288:156-161.

18. Kuo KT, Wang HW, Chou TY, Hsu WH, Hsu HS, Lin CH, Wang LS: Prognostic role of PGE2 receptor EP2 in esophageal squamous cell carcinoma. Ann Surg Oncol 2009, 16:352-360.

19. Yang J, Liu X, Bhalla K, Kim CN, Ibrado AM, Cai J, Peng TI, Jones DP, Wang X: Prevention of apoptosis by $\mathrm{Bcl}-2$ : release of cytochrome $\mathrm{c}$ from mitochondria blocked. Science 1997, 275:1129-1132.

20. Manon S, Chaudhuri B, Guérin M: Release of cytochrome $c$ and decrease of cytochrome c oxidase in Bax-expressing yeast cells, and prevention of these effects by coexpression of Bcl-xL. FEBS Lett 1997, 415:29-32.

21. Oyama T, Osaki T, Baba T, Nagata Y, Mizukami M, So T, Nakata S, Ichiki Y, Uramoto H, Sugaya M, Yoshimatsu T, Morita M, Hanagiri T, Sugio K, Kawamoto T, Yasumoto K: Molecular genetic tumor markers in non-small cell lung cancer. Anticaner Res 2005, 25:1193-1196.

22. Massion PP, Carbone DP: The molecular basis of lung cancer: molecular abnormalities and therapeutic implications. Respir Res 2003, 4:12.

23. Johnson DH, Fehrenbacher L, Novotny WF, Herbst RS, Nemunaitis Jנ, Jablons DM, Langer CJ, DeVore RF 3rd, Gaudreault J, Damico LA, Holmgren E, Kabbinavar F: Randomized phase II Trial comparing bevacizumab plus carboplatin and paclitaxel with carboplatin and paclitaxel alone in previously untreated locally advanced or metastatic non-small-cell lung cancer. J Clin Oncol 2004, 22:2184-2191.

24. Sandler A, Gray R, Perry MC, Brahmer J, Schiller JH, Dowlati A, Lilenbaum R, Johnson DH: Paclitaxel-carboplatin alone or with bevacizumab for nonsmall-cell lung cancer. N Engl J Med 2006, 355:2542-2550.

25. Reck M, von Pawel J, Zatloukal P, Ramlau R, Gorbounova V, Hirsh V, Leighl N, Mezger J, Archer V, Moore N, Manegold C: Phase III trial of cisplatin plus gemcitabine with either placebo or bevacizumab as first-line therapy for nonsquamous non-small-cell lung cancer: AVAil. J Clin Oncol 2009, 27:1227-1234.

26. Littlepage LE, Sternlicht MD, Rougier N, Phillips J, Gallo E, Yu Y, Williams K, Brenot A, Gordon Jl, Werb Z: Matrix metalloproteinases contribute distinct roles in neuroendocrine prostate carcinogenesis, metastasis, and angiogenesis progression. Cancer Res 2010, 70:2224-2234.

27. Park JK, Park SH, So K, Bae IH, Yoo YD, Um HD: ICAM-3 enhances the migratory and invasive potential of human non-small cell lung cancer cells by inducing MMP-2 and MMP-9 via Akt and CREB. Int J Oncol 2010, 36:181-192.

28. Merkle M, Ribeiro A, Sauter M, Ladurner R, Mussack T, Sitter T, Wörnle M: Effect of activation of viral receptors on the gelatinases MMP-2 and MMP-9 in human mesothelial cells. Matrix Biol 2010, 29:202-208.

29. Fornuskova D, Stiburek L, Wenchich L, Vinsova K, Hansikova H, Zeman J: Novel insights into the assembly and function of human nuclearencoded cytochrome coxidase subunits $4,5 \mathrm{a}, 6 \mathrm{a}, 7 \mathrm{a}$ and $7 \mathrm{~b}$. Biochem $\mathrm{J}$ 2010, 428:363-374. 
30. Lenka N, Vijayasarathy C, Mullick J, Avadhani NG: Structural organization and transcription regulation of nuclear genes encoding the mammalian cytochrome coxidase complex. Prog Nucleic Acid Res Mol Biol 1998, 61:309-344.

31. Ludwig B, Bender E, Arnold S, Hüttemann M, Lee I, Kadenbach B: Cytochrome $\mathrm{C}$ oxidase and the regulation of oxidative phosphorylation Chembiochem 2001, 2:392-403.

32. Brzezinski P, Johansson AL: Variable proton-pumping stoichiometry in structural variants of cytochrome c oxidase. Biochim Biophys Acta 2010, 1797:710-723

33. Chen $Z X$, Pervaiz $\mathrm{S}: \mathrm{BCl}-2$ induces pro-oxidant state by engaging mitochondrial respiration in tumor cells. Cell Death Differ 2007, 14:1617-1627.

34. Chen ZX, Pervaiz S: Involvement of cytochrome c oxidase subunits Va and $\mathrm{Vb}$ in the regulation of cancer cell metabolism by Bcl-2. Cell Death Differ 2010, 17:408-420.

35. Choi J, Choi K, Benveniste EN, Rho SB, Hong YS, Lee JH, Kim J, Park K: BCl-2 promotes invasion and lung metastasis by inducing matrix metalloproteinase-2. Cancer Res 2005, 65:5554-5560.

36. Noujaim D, van Golen CM, van Golen KL, Grauman A, Feldman EL: N-Myc and $\mathrm{BCl}-2$ coexpression induces MMP-2 secretion and activation in human neuroblastoma cells. Oncogene 2002, 21:4549-4557.

37. Nieto N, Rojkind M: Repeated whiskey binges promote liver injury in rats fed a choline-deficient diet. J Hepatol 2007, 46:330-339.

doi:10.1186/1471-2407-12-273

Cite this article as: Chen et al:: The role of cytochrome $c$ oxidase subunit Va in non-small cell lung carcinoma cells: association with migration, invasion and prediction of distant metastasis. BMC Cancer 2012 12:273.

\section{Submit your next manuscript to BioMed Central and take full advantage of:}

- Convenient online submission

- Thorough peer review

- No space constraints or color figure charges

- Immediate publication on acceptance

- Inclusion in PubMed, CAS, Scopus and Google Scholar

- Research which is freely available for redistribution 